\title{
Joint position sense and vibration sense: anatomical organisation and assessment
}

\section{S Gilman}

J Neurol Neurosurg Psychiatry 2002;73:473-477

Clinical examination of joint position sense and vibration sense can provide important information concerning specific cutaneous sensory receptors, peripheral nerves, dorsal roots, and central nervous system pathways and should be included as a regular component of the neurological examination. Although these sensory modalities share a spinal cord and brainstem pathway, they arise in different receptors and terminate in separate distributions within the thalamus and cerebral cortex. Consequently, both modalities should be tested as part of the neurological examination. Clinical testing of these modalities requires simultaneous stimulation of tactile receptors; hence this review will include information about the receptors and pathways responsible for tactile sensation.

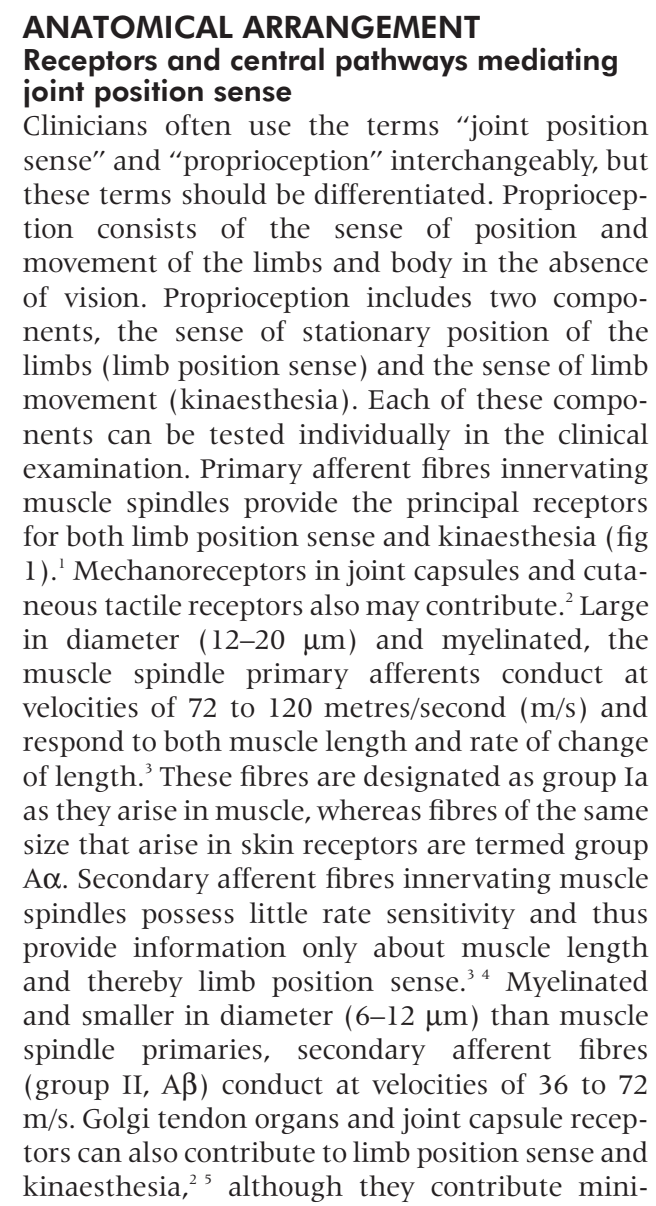

mally to joint position sense as clinically tested. ${ }^{1}$ Golgi tendon organs consist of receptors in tendons that mediate contractile force or effort from a group of muscle fibres. ${ }^{3}$ Myelinated and large in diameter $(12-20 \mu \mathrm{m})$, the afferent fibres of Golgi tendon organs (group Ib, A $\alpha$ ) conduct at velocities equal to those of muscle spindle primary afferents. Joint capsules receive innervation principally from small afferent fibres. These include thinly myelinated group III (A $\delta)$ fibres, which have diameters of $1-6 \mu \mathrm{m}$ and conduct at 4 to $36 \mathrm{~m} / \mathrm{s}$, and unmyelinated group IV (C) fibres, which have diameters of $0.2-1.5 \mu \mathrm{m}$ and conduct at $0.4-2.0 \mathrm{~m} / \mathrm{s}$. Most of these afferents are nociceptors that respond to the extremes of joint position; however, joint capsules also receive some innervation from mechanoreceptors that respond to joint angle. ${ }^{2}$ The afferent fibres of these receptors consist of myelinated group II $(\mathrm{A} \beta)$ fibres with diameters of $6-12 \mu \mathrm{m}$ and conduction velocities of 36 to $72 \mathrm{~m} / \mathrm{s}$. Finally, as described further below, cutaneous afferent fibres activated by skin stretch also contribute to the sense of movement and direction of movement.

The afferent fibres mediating joint position sense and kinaesthesia course through peripheral nerves into the medial aspect of the dorsal roots and then enter the dorsal horn of the spinal cord. Many of these afferents form synaptic connections with second order neurons in the deeper layers of the dorsal horn, and second order neurons ascend through the ipsilateral dorsolateral funiculus of the spinal cord (fig 1). Fibres ascending the dorsolateral funiculus form synaptic connections with the lateral cervical nucleus, which is located in the two upper cervical segments of the spinal cord, immediately ventral to the dorsal horn. Postsynaptic neurons from the lateral cervical nucleus project across the midline of the spinal cord, ascend to enter the medulla, and join the medial lemniscus (fig 1). Currently it appears that the dorsolateral funiculus is the principal ascending spinal cord pathway for joint position sense and kinaesthesia. ${ }^{16}$ Some afferents mediating joint position sense and kinaesthesia project directly into the ipsilateral dorsal columns and ascend the spinal cord, terminating in the dorsal column nuclei. The dorsal columns mediate only discrimination of the frequency and duration of repetitive tactile stimuli, as this function becomes impaired after dorsal column transection in experimental animals..$^{7-9}$ Fibres ascending the dorsal columns develop a topographical arrangement. Afferent fibres join the lateral aspect of the dorsal columns in succession, caudal to rostral, at each spinal cord segment. This causes a laminated pattern, with fibres from the more caudal segments positioned medially 


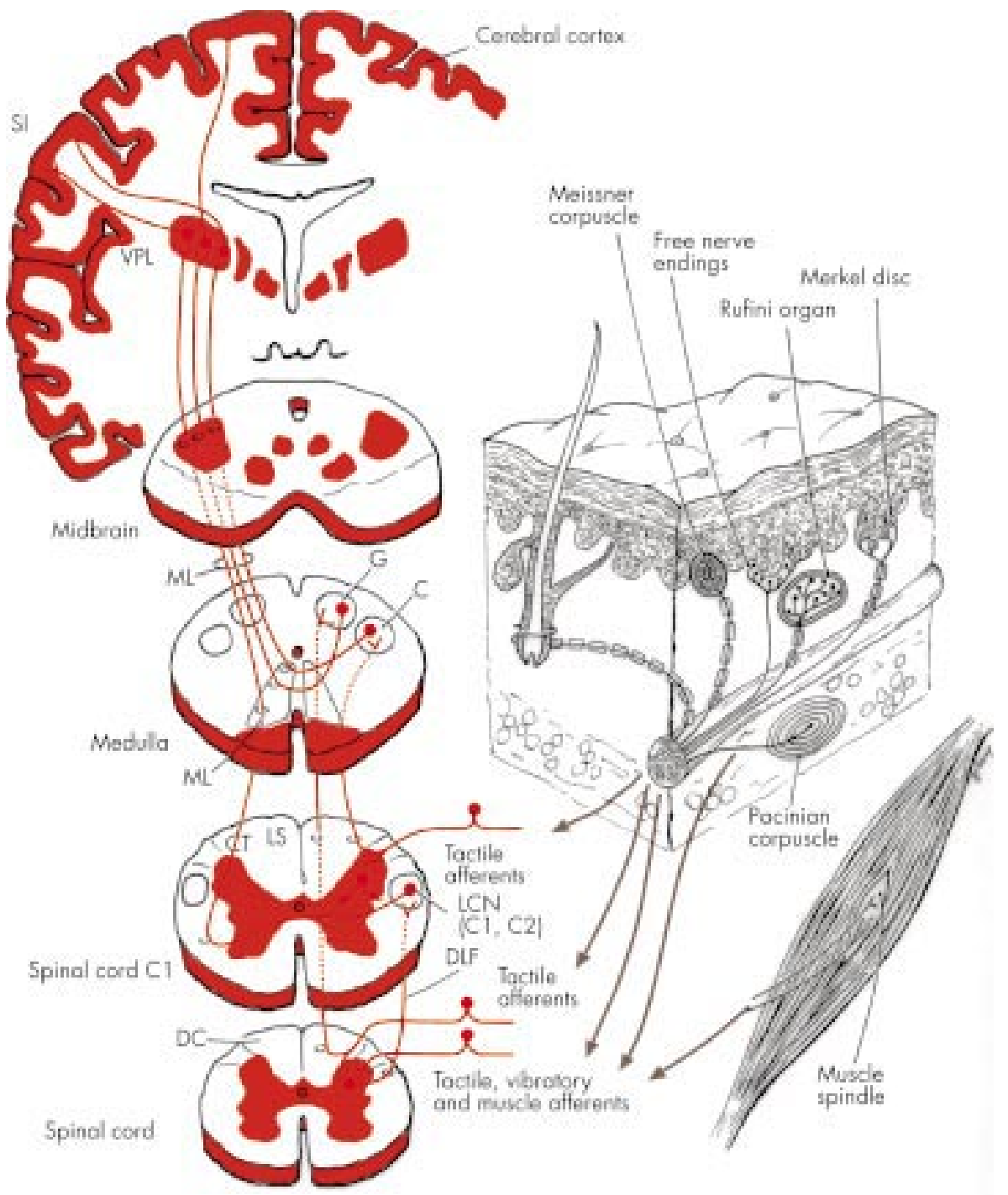

Figure 1 Diagram of the peripheral receptors and central pathways mediating joint position sense, vibration sense, and tactile sensation. The lower diagram on the right illustrates the receptors principally responsible for position sense, which are muscle spindle primary and secondary afferents. The upper diagram on the right illustrates the location and morphology of mechanoreceptors in glabrous (hairless) and hairy skin of the human hand. The receptors are located both in the superficial skin at the junction of dermis and epidermis and in the deeper dermis and subcutaneous tissue. Glabrous skin contains Meissner's corpuscles, located in dermal papillae; Merkel disc receptors, located between dermal papillae; and free nerve endings. Hairy skin contains hair receptors, Merkel's receptors and free nerve endings. Subcutaneous receptors located in both glabrous and hairy skin include pacinian corpuscles and Ruffini endings. Merkel disc receptors, Meissner's corpuscles and pacinian corpuscles are capable of mediating vibration sense, but pacinian corpuscles are responsible for detection of vibration as tested clinically. Multiple receptors mediate tactile sensation, including Meissner's corpuscles, Merkel discs, Ruffini endings, pacinian corpuscles, and hair follicle receptors. The diagram on the left illustrates the central pathways mediating joint position sense, vibration sense, and tactile sensation. Afferent fibres innervating pacinian corpuscles, muscle spindles, and tactile receptors make synaptic connections with dorsal horn neurons that project rostrally through the dorsolateral funiculus (DLF) and terminate in the lateral cervical nucleus (LCN) at spinal cord segments $\mathrm{C} 1$ and C2. Fibres from the LCN project across the midline and ascend into the medulla, where they join the medial lemniscus. Some afferent fibres innervating tactile receptors bifurcate in the dorsal horn, with one branch entering the dorsal columns (DC) and the other making a synaptic connection on dorsal horn neurons with axons that cross the midline and project through the lateral spinothalamic tract (not shown in the diagram) or the DLF. Fibres in the DC are laminated, with those from the sacral region (S) most medial, and lumbar (L), thoracic (T), and cervical (C) sequentially more lateral. DC fibres from sacral and lumbar segments terminate in the gracile (G) nucleus and fibres from thoracic and cervical segments terminate in the cuneate $(C)$ nucleus of the medulla. Fibres projecting from the $G$ and $C$ nuclei pass across the midline and enter the medial lemniscus, which ascends to the ventral posterior lateral (VPL) nucleus of the thalamus. Thalamocortical fibres from VPL project to the primary somatosensory cortex $(\mathrm{S} 1)$ of the postcentral gyrus.

and fibres from the rostral segments more laterally. In the upper spinal cord, fibres arising in sacral, lumbar and lower thoracic segments form the medial (gracile) fascicle and those arising in the upper thoracic and cervical segments form the lateral (cuneate) fascicle. Most fibres conveying joint position sense and kinaesthesia from the trunk and upper limbs that enter the cuneate fascicle run their full length up to the medulla in this structure. In contrast, most of the fibres conveying joint position sense and kinaesthesia from the lower extremities depart from the gracile fascicle in the upper lumbar cord and terminates upon neurons of Clarke's column. ${ }^{10}$ Neurons of Clarke's column project to nucleus Z in the medulla and neurons from this nucleus project into the medial lemniscus with proprioceptive fibres from the cuneate nucleus. The fibres remaining in the gracile fascicle contain principally those conveying tactile sensation. Afferents ascending the dorsal columns make synaptic connection in the dorsal column nuclei of the medulla. The medial branches 
from the fasciculus gracilis terminate in the nucleus gracilis and the lateral branches form the fasciculus cuneatus and terminate in the nucleus cuneatus. Axons of neurons arising in the gracile and cuneate nuclei form the medial lemniscus, which crosses the midline and receives fibres from the lateral cervical nucleus (fig l) and nucleus $\mathrm{Z}$ (not illustrated). The medial lemniscus then ascends the brainstem to terminate in the ventral posterolateral (VPL) nucleus of the thalamus (fig 1). Afferents mediating joint position sense and kinaesthesia from the face project to the principal trigeminal nucleus, and postsynaptic neurons send axons rostrally through the trigeminal lemniscus. The trigeminal lemniscus moves upward through the brainstem in parallel to the medial lemniscus, terminating in the ventral posteromedial (VPM) nucleus of the thalamus. Thalamocortical projections from the VPL and VPM ascend to the primary somatosensory areas (S1) of the postcentral gyrus of the cerebral cortex, terminating upon cerebral cortical neurons that encode movement and position (fig 1). ${ }^{11}$ The somatotopic organisation of the afferents remains preserved throughout the entire ascending pathway from the spinal cord to the somatosensory cortex. Currently it appears that the dorsolateral funiculus is the principal ascending spinal cord pathway for joint position sense and kinaesthesia. $^{1}$

\section{Receptors and central pathways mediating vibration sense}

The sense of vibration results from the sinusoidal oscillation of objects placed against the skin. Mechanoreceptors in the skin respond to the oscillations by developing action potentials that are transmitted through their neural afferents, with a pulse code in which each action potential signals one cycle of a sinusoidal wave. The frequency of action potentials in the afferent nerve fibre signals the vibration frequency. The perception of vibration as a series of rapidly repeating sensation results from the simultaneous activation of multiple receptors, leading to synchronous discharge among many afferent fibres. The receptors responsible for vibration sense include Merkel disk receptors and Meissner's corpuscles in the superficial layers of the skin and pacinian corpuscles in deeper layers of skin, between layers of muscle, and in periosteum (fig 1). The receptor afferents are myelinated and include both large diameter (group $\mathrm{A} \alpha$, diameter 12-20 $\mu \mathrm{m}$, conduction velocities 72 to $120 \mathrm{~m} / \mathrm{s}$ ) and medium diameter (group $A \beta$, diameter $6-12 \mu \mathrm{m}$, conduction velocities 36 to $72 \mathrm{~m} / \mathrm{s}$ ) fibres. Merkel disk receptors respond maximally to low frequencies (5-15 Hz), Meissner's corpuscles to mid-range frequencies (20-50 Hz), and pacinian corpuscles to high frequencies (60$400 \mathrm{~Hz}$ ). Receptor tuning thresholds determine the capacity to detect vibration. The total number of sensory nerve fibres activated by a vibrating stimulus determines the intensity of vibration; the frequency of firing determines the vibration frequency perceived. Humans are most responsive to vibration at frequencies of $200-250 \mathrm{~Hz}^{2}$

Fibres mediating vibration sense course through peripheral nerves and dorsal roots to enter the spinal cord. These fibres bifurcate, with one branch terminating upon neurons in the deeper layers of the dorsal horn and the other entering the dorsal columns (fig 1). Second order neurons from the dorsal horn ascend through the ipsilateral dorsolateral funiculus, terminating upon neurons in the lateral cervical nucleus. Postsynaptic neurons from the lateral cervical nucleus project across the midline of the spinal cord, ascend to enter the medulla, and join the medial lemniscus (fig 1). The dorsolateral pathway appears to be the important one mediating vibration sense in the human. ${ }^{1}$ The dorsal root collaterals entering the dorsal columns ascend the spinal cord ipsilaterally, terminating in the dorsal column nuclei of the medulla. Fibres of the dorsal columns maintain a topographical arrangement, with those from the caudal segments positioned medially and those from the rostral segments laterally. The medially placed fibres form synapses in the gracile nucleus and the laterally placed fibres form synapses in the cuneate nuclei. Neurons emanating from the gracile and cuneate nuclei form the medial lemniscus, which crosses the midline and receives fibres from the lateral cervical nucleus (fig l). The medial lemniscus then ascends the brainstem to terminate in the VPL nucleus of the thalamus. Afferents mediating vibration sense from the face project to the principal trigeminal nucleus, and postsynaptic neurons send fibres through the trigeminal lemniscus to terminate in the VPM nucleus of the thalamus. Thalamocortical projections from the VPL and VPM ascend to the primary somatosensory areas (S1) of the postcentral gyrus of the cerebral cortex, terminating upon vibratory responsive neurons ( fig 1). ${ }^{12}{ }^{13}$ The VPL, VPM, and S1 contain detailed somatotopic maps of the body.

\section{Differences in the central pathways mediating joint} position sense and vibration sense

Although the central pathways mediating joint position sense and vibration sense appear to be identical, they terminate upon different thalamic and cerebral cortical neurons. Moreover, different receptors mediate these sensory functions. Some neurological disorders affect one of these sensory functions while partially or completely sparing the other.

\section{Receptors and central pathways mediating tactile sensation}

During the clinical examination of the nervous system it is impossible to avoid stimulating tactile afferents when testing joint position sense no matter how delicately the examiner conducts the evaluation. Tactile sensitivity is greatest on the glabrous (hairless) skin of the hands, the soles of the feet, and the lips. Four mechanoreceptors have been identified in glabrous skin, two in the superficial layers (Meissner's corpuscles and Merkel discs) and two in the subcutaneous tissue (Ruffini endings and pacinian corpuscles) (fig 1). Meissner's corpuscles are rapidly adapting mechanoreceptors, meaning that they respond only during the onset of skin stimulation. Meissner's corpuscles respond to stroking and fluttering types of tactile stimuli. Merkel disc receptors are slowly adapting receptors, responding at constant discharge rates during constant intensity stimulation. Merkel disc receptors respond to pressure and texture. As described above, pacinian corpuscles are rapidly adapting and respond to vibration. Ruffini endings are slowly adapting receptors that respond to skin stretch. These endings link subcutaneous tissue to skin folds overlying joints. The afferent fibres of all of these receptors afferents are myelinated and include both large diameter (group $\mathrm{A} \alpha$, diameter $12-20 \mu \mathrm{m}$, conduction velocities 72 to $120 \mathrm{~m} / \mathrm{s}$ ) and medium diameter (group $\mathrm{A} \beta$, diameter $6-12 \mu \mathrm{m}$, conduction velocities 36 to $72 \mathrm{~m} / \mathrm{s}$ ) fibres. Hairy skin contains similar mechanoreceptors, but includes hair follicle receptors and field receptors, which are the principal rapidly adapting receptors (fig 1). Hair follicle receptors respond to hair displacement and field receptors sense skin stretch with joint movement or rubbing of the skin. The afferent fibres of these receptors are myelinated and include large (group $A \alpha)$, medium (group $A \beta$ ), and small (group $\mathrm{A} \delta$ ) diameter fibres.

Diseases of the central nervous system frequently spare tactile sensation because many pathways carry tactile information to the brain. Tactile afferents course through peripheral nerves to dorsal roots and enter the dorsal part of the spinal cord through the medial portions of the dorsal roots. One branch of each afferent fibre enters the dorsal column and ascends the ipsilateral spinal cord in a somatotopic arrangement, making synaptic connection with the dorsal column nuclei of the medulla (fig 1). As mentioned above, transection of the dorsal columns does not affect the ability to detect tactile sensation or tactile movements, but impairs discrimination of the frequency and duration of repetitive tactile stimuli 
and thus the ability to determine the direction of movement of cutaneous stimuli. ${ }^{14}$ Postsynaptic neurons in the dorsal column nuclei form the medial lemniscus, which crosses the midline and terminates in the VPL nucleus of the thalamus (fig 1). The second branch of each afferent fibre entering the dorsal spinal cord forms a synaptic connection with neurons in the dorsal horn. Second order neurons ascend the spinal cord either ipsilaterally or contralaterally. Those travelling ipsilaterally course through the dorsolateral white matter of the spinal cord to the lateral cervical nucleus, where they form synaptic connections. Postsynaptic neurons from the lateral cervical nucleus cross the midline and enter the medial lemniscus, terminating in the VPL nucleus. Second order neurons originating in the dorsal horn that ascend contralaterally cross the midline within two to four spinal segments and enter the lateral spinothalamic projections to the lateral and medial thalamus. Transection of the lateral spinothalamic tract does not affect tactile threshold or discriminative capacity, but causes loss of tactile erogenous sensations caudal and contralateral to the lesion. ${ }^{1}$ The thalamus sends projections conveying tactile information to the primary (S1) somatosensory cortex of the postcentral gyrus (fig 1). ${ }^{11}{ }^{16}$ The somatotopic map conveyed from peripheral receptors becomes more elaborate in the primary somatosensory cortex, particularly for cutaneous surfaces used in exploration of the environment such as the lips, tongue, and finger pads. Many neurons in the primary somatosensory cortex respond most rapidly in response to movement of a cutaneous stimulus in a particular direction. Hence if peripheral and subcortical pathways are intact, impairment of cutaneous direction sense suggests disease within the postcentral cortex. Neurons in the somatosensory cortex project to the posterior parietal association areas, where tactile information becomes integrated with other sensory information. ${ }^{16}{ }^{17}$

\section{Assessment of joint position sense and vibration sense}

The history frequently provides clues to the type of sensory disturbance the clinician will encounter on examination. Sensory symptoms such as pain and paraesthesias, tingling, pins and needles, or numbness frequently result from involvement of pain and temperature sensations and therefore the smaller diameter fibres and not the fibres mediating position sense or vibration sense. In contrast, clues to abnormalities of position sense or vibration sense come from complaints of clumsiness in walking, incoordination of the upper extremities, frequent stumbling, and difficulty standing upright with the eyes closed, as in washing the face in the shower or at a sink. Involuntary movements (pseudoathetosis) at rest or with the limbs outstretched and the eyes closed also suggest abnormalities of these sensory modalities.

In testing position sense clinically, ask your patient to look at the great toe while you demonstrate the positions of dorsiflexion and ventral flexion (up and down). Then lightly grasp the great toe on its medial and lateral surfaces between your first finger and thumb, move the digit up, hold it steady and ask the patient with eyes closed to indicate whether the toe has been moved up or down. Move the joint slowly, preferably over one to two seconds, as the sensitivity of the test is inversely related to the velocity of the displacement. It is important to hold the digit laterally, parallel to the plane of movement, to avoid stimulation of tactile pressure receptors that may give clues to the direction of movement. Normally, subjects can correctly identify movement of one degree or less across the joints of the fingers and movement of three degrees at the toes. If position sense appears to be impaired at the great toe, test position sense at the ankle by grasping the foot on its medial and lateral surfaces between your thumb and first finger, repeating the same type of movement. If position sense is impaired there, test position sense successively at the knee and, if abnormal, test at the hip. It becomes difficult to avoid large tactile contact when testing movement about the knee and hip. Move on to the upper extremities if abnormalities are found in the lower extremities. Joint position sense can be evaluated with static stimuli, as described above, or with dynamic stimuli. For the latter, ask the patient to determine the direction of movement as you move the digit. I find it less confusing for the patient to test static position initially and then attempt dynamic stimuli if impairment appears. Most patients who have defective position sense in the great toe but preserved position sense in the ankle and more proximal components of the limb can stand eyes open with feet together and remain upright with the eyes closed (negative Romberg sign). Patients with abnormalities of joint position sense at the toes, ankes, and knees, and particularly at the hips, have a positive Romberg sign.

Begin testing vibration sense by applying the base of a vibrating 128 CPS tuning fork to the great toe and ask the patient to describe the sensation. It is best to test patients with their eyes closed and ask them to determine whether the tuning fork is vibrating or not. Apply the vibrating tuning fork to skin overlying bone instead of skin over fat or muscle so that the vibration will be transmitted through a large volume of tissue. Nevertheless, the stimulus can be applied to the skin in any portion of the body, as pacinian corpuscles are located widely throughout the body. If the patient cannot determine whether the tuning fork is vibrating, test the patient at the medial or lateral malleolus, and move successively higher along the body if you find abnormalities distally. In the elderly population, particularly those over 70 years of age, vibration sense may be absent at the great toe, but should be preserved at the medial or lateral malleolus. Initially ask the patient to determine only whether the tuning fork is vibrating or not. For more detailed testing, the force used to strike the tines of the tuning fork can be decreased to apply a progressively weaker stimulus until the patient no longer detects the stimulus. You can assess the patient's threshold against your own by applying the vibrating tuning fork to your finger when the stimulus has dropped below the patient's threshold.

\section{Disorders affecting position sense and vibration sense}

Diseases of the sensory nerves can affect primarily large myelinated fibres, thereby affecting position sense and vibration sense with relatively little impairment in the detection of tactile or noxious stimuli. These are frequently the acute or demyelinating polyneuropathies, but neurotoxins and nutritional neuropathies also can impair vibration sense or position sense, although these disorders commonly affect tactile sensation as well. Radiculopathies and plexopathies usually affect both the large myelinated fibres mediating position sense and vibration sense and the small, thinly myelinated (group $\mathrm{A} \delta$ ) or unmyelinated (group $\mathrm{C}$ fibres) nerve fibres mediating temperature and pinprick sensations. Accordingly, it is rare that a radiculopathy affects position sense or vibration sense with preservation of nociceptive sensations.

Spinal cord lesions usually present with segmental demarcation of sensory abnormalities with a sensory (and often a motor) level, including diminished sensation and an upper motor neuron type of weakness below the level, with preservation of these functions above the level. As indicated above, disease selectively affecting the dorsal columns does not impair position sense and vibration sense. In contrast, disorders affecting the dorsolateral portions of the spinal cord do selectively impair these senses, usually with preservation of tactile and nociceptive sensation. The association of a unilateral upper motor neuron type of weakness with impairment of position sense and vibration sense in the ipsilateral limbs and impaired pain and temperature sensation in the opposite limbs points to a Brown-Séquard syndrome because of the disease affecting the dorsolateral and lateral regions of the spinal cord unilaterally. Occlusion of the anterior spinal 
artery can cause a selective and usually bilateral decrease of pain and temperature sensation. In contrast, infarction in the territory of the dorsal or radicular spinal arteries, though infrequent, can impair ipsilateral joint position sense and vibration sense. Neurofibromas, schwannomas and other tumours of the dorsal roots that compress the dorsolateral spinal cord can cause both a radicular and a segmental sensory deficit, with impairment of pain or tactile sensation ipsilaterally in the root distribution. Below the level of the tumour, position sense and vibration sense are impaired ipsilaterally and pain and temperature are impaired contralaterally. Descending motor systems can be spared; hence this location of disease may not evoke the full Brown-Séquard syndrome.

Brainstem lesions commonly resemble those found with spinal cord disease because of selective impairment of spinothalamic tract or medial lemniscus function. Disease involving the medial portions of the medulla affects the medial lemniscus, causing contralateral loss of position sense and vibration sense without loss of pain or temperature sensation. As the medial lemniscus of the left and right sides lie immediately adjacent at lower brainstem levels, impairment of position sense and vibration sense often occurs bilaterally. Moreover, the corticospinal tract may become affected with medial and ventral medullary lesions, as this structure lies immediately ventral to the medial lemniscus. Accordingly, diseases affecting the medial and ventral portions of the medulla may cause upper motor neuron weakness accompanying the sensory disturbances. Disorders affecting the dorsolateral regions of the medulla or pons cause loss of pain and temperature sensation while sparing position sense, vibration sense and tactile sensation. In contrast, lateral medullary or pontine lesions spare the corticospinal tracts; hence upper motor neuron weakness does not accompany these lesions. Involvement of vestibular and cerebellar connections and autonomic nuclei of the dorsolateral medulla and pons induce additional symptoms.

Diseases affecting the thalamus frequently impair both spinothalamic and lemniscal functioning. Accordingly, large lesions of the ventrolateral and ventromedial thalamus result in complete loss of somatic sensation on the contralateral side of the body, including pain, thermal sensation, tactile sensation, position sense, and vibration sense. Lesser lesions of the thalamus can affect these sensory functions in any combination.

Disease of the primary somatosensory cortex does not usually produce as pronounced sensory symptoms as lesions at the thalamic level. Frequently the patient has no sensory complaint, but experiences difficulty performing fine or delicate manipulations that depend upon tactile and position sense information. When sensory symptoms occur, they can involve any part of the contralateral side of the body, depending upon the location of the lesion. Lesions at the cerebral cortical level rarely cause loss of primary sensory modalities. Disease at this level results in loss of discriminative functions such as the direction of a moving tactile stimulus on the skin, identifying two points applied simultaneously to the skin in close spatial approximation, and determining the direction of movement about the interphalangeal joints, which is a kinaesthetic sense.

Testing vibration sense can be helpful in detecting psychogenic sensory disorders. Some patients complain of a marked decrease or total absence of all sensation on one half of the body. Others may state that they have a marked decrease or loss of specific modalities of sensation, frequently tactile sensation. These patients often complain of associated muscle weakness, but on testing the weakness proves to be a give-way type of weakness, in which an initially forceful muscle contraction suddenly lets go. Usually the muscle stretch reflexes show no abnormalities and the plantar response remains flexor on the affected side. It is helpful to test vibration sense on the forehead or on the sternum in patients with this constellation of findings. A vibrating tuning fork activates pacinian corpuscles very rapidly and widely across the periosteum of the skull or the sternum. Consequently, patients who claim that they can feel a vibrating tuning fork near the midline of the forehead or the sternum but have either markedly decreased or absent sensation when the vibrating tuning fork is placed on the forehead or sternum only a few millimetres beyond the midline on the opposite side are likely to have psychogenic symptoms. This finding is sometimes termed "splitting the sternum" or "splitting the forehead" for vibration sense.

\section{Author's affiliation}

Dr S Gilman, Department of Neurology, University of Michigan Medical Center, 1500 E Medical Center Drive, 1914 Taubman Center, Ann Arbor Ml 48109-0316, USA

Competing interests: none declared

\section{REFERENCES}

1 Casey KL. The somatosensory system. In: Gilman S, ed. Clinical examination of the nervous system. New York: McGraw-Hill, 1999: 175-211.

2 Gardner EP, Martin JH, Jessell TM. The bodily senses. In: Kandel ER, Schwartz JH, Jessell TM, eds. Principles of neural science. New York: McGraw-Hill, 2000:430-50

3 Prochazka A, Gorassini M. Ensemble firing of muscle afferents recorded during normal locomotion in cats. J Physiol 1998;507:293-304.

4 Nardone A, Tarantola J, Miscio G, et al. Loss of large-diameter spindle afferent fibres is not detrimental to the control of body sway during upright stance: evidence from neuropathy. Exp Brain Res 2000; 135: 155-62

5 Prochazka A. Proprioceptive feedback and movement regulation. In: Rowell L, Sheperd JT, eds. Handbook of physiology: regulation and integration of multiple systems. New York: American Physiological Society, 1996:89-127.

6 Glendinning DS, Vierck CJ Jr. Lack of a proprioceptive deficit after dorsal column lesions in monkeys. Neurology 1993;43:363-6.

7 Vierck CJ Jr, Cooper BY. Cutaneous texture discrimination following transection of the dorsal spinal column in monkeys. Somatosens Mot Res 1998; 15:309-15.

8 Vierck CJ Jr. Impaired detection of repetitive stimulation following interruption of the dorsal spinal column in monkeys. Somatosens Mot Res 1998;15:157-63.

9 Makous JC, Friedman RM, Vierck CJ Jr. Effects of a dorsal column lesion on temporal processing within the somatosensory system of primates. Exp Brain Res 1966;112:253-67.

10 Webster KE. Somaesthetic pathways. Br Med Bull 1977;33:1 13-20.

11 Debowy DJ, Ghosh S, Ro JY, et al. Comparison of neuronal firing rates in somatosensory and posterior parietal cortex during prehension. Exp Brain Res 2001;137:269-91.

12 Willis WD. The pain system: the neural basis of nociceptive transmission in the mammalian nervous system. Basel: Karger, 1985

13 Tommerdahl $M$, Delemos KA, Favorov OV, et al. Response of anterior parietal cortex to different modes of same-site skin stimulation. $J$ Neurophysiol 1998:80:3272-83.

14 Makous JC, Vierck CJ Jr. Physiological changes during recovery from a primate dorsal column lesion. Somatosens Mot Res 1994;11:183-92.

15 Tommerdahl M, Delemos KA, Vierck CJ Jr, et al. Anterior parietal cortical response to tactile and skin-heating stimuli applied to the same skin site. J Neurophysiol 1996;75:2662-70.

16 Ro JY, Debowy D, Ghosh S, et al. Depression of neuronal firing rates in somatosensory and posterior parietal cortex during object acquisition in a prehension task. Exp Brain Res 2000;135:1-11.

17 Gardner EP, Ro JY, Debowy D, et al. Facilitation of neuronal activity in somatosensory and posterior parietal cortex during prehension. Exp Brain Res 1999;127:329-54 